\title{
Página web para fortalecer el conocimiento general de la física: un ejemplo de proyecto semillero
}

\section{Web page to strengthen the general knowledge of physics: an example of a seed project}

Ing. Christian Pavón Brito, Msc.

Universidad de Guayaquil, Ecuador

Ing. Jorge Encalada Noboa, Msc.

Universidad de Guayaquil, Ecuador

Kevin Matute Fernández

Universidad de Guayaquil, Ecuador

Autor para correspondencia: jorge.encaladan@ug.edu.ec, christian.pavonb@ug.edu.ec

Fecha de recepción: 10 de Septiembre de 2016 - Fecha de aceptación: 01 de Noviembre de 2016

Resumen: En el presente trabajo se muestra un ejemplo de un proyecto semillero que surgió en un aula de un curso de Física de una universidad del Ecuador. Los participantes en el proyecto semillero fueron seis estudiantes de un curso de tercer semestre: dos mujeres y cuatro hombres entre 19 y 22 años de edad, en conjunto con un profesor tutor. Se detalla el proceso de elaboración del proyecto en el aula de clase durante tres sesiones y se presenta en la parte de resultados las partes claves del mismo, como el objetivo general, los objetivos específicos, el impacto y los resultados esperados cuando el proyecto finalice. Se espera que este trabajo inspire a más profesores para llevar el aprendizaje basado en proyectos a su clase, y que proporcione ideas, consejos y herramientas para involucrar a sus estudiantes.

Palabras claves: aprendizaje basado en proyectos; proyecto semillero; proyecto de aula; estrategia instruccional

\begin{abstract}
The present paper shows an example of a seed project that emerged in a Physics classroom of an university of Ecuador. The participants in the seed project were six students in a course of third semester: two women and four men between 19 and 22 years old, together with a tutor. It is detailed the process of development of the project in the classroom during three sessions and it is presented in the results the key parts as the overall objective, specific objectives, the impact and the expected results when the project ends. It is hoped that this work will inspire more teachers to bring project based learning to class, and provides ideas, tips and tools to engage their students.
\end{abstract}

Key words: project based learning; seed project; classroom project; instructional strategy 


\section{Introducción}

La introducción de proyectos en el plan de estudios no es una idea nueva o revolucionaria en la educación. No obstante, durante la última década esta se ha convertido en una estrategia de enseñanza más formal.

El aprendizaje basado en proyectos ha ganado un mayor punto de apoyo en el aula ya que los investigadores han documentado lo que los profesores han entendido desde hace tiempo: los estudiantes se vuelven más comprometidos en el aprendizaje cuando tienen una oportunidad de profundizar en el complejo, desafiante, problemático y a veces confuso terreno de los proyectos, muchos de los cuales se parecen a la vida real.

Sin embargo, el aprendizaje basado en proyectos va más allá de generar el interés en los estudiantes. Los proyectos bien diseñados alientan una indagación activa y un pensamiento de orden superior (Thomas, 1998). Las habilidades de los estudiantes para adquirir nuevos conocimientos se incrementan cuando son vinculados a actividades significativas (Bransford, Brown \& Cocking, 2000), también cuando se les ayuda a entender el por qué, cuándo y cómo esos hechos y habilidades son relevantes.

Otra ventaja dentro del contexto de la educación, es que la investigación adquiere un significado más específico. Los maestros que utilizan la investigación como estrategia normalmente motivan a que los estudiantes realicen preguntas, planeen y lleven a cabo investigaciones, hagan observaciones, y reflexionen sobre lo que han descubierto. Eso no quiere decir que esta sea una definición estática. Incluso dentro de una misma clase, las actividades de investigación pueden surgir tanto del profesor como de los estudiantes (Jarrett, 1997).

Para los estudiantes, los beneficios del aprendizaje basado en proyectos incluyen: aumento de la autogestión y mejora de las actitudes hacia el aprendizaje (Thomas, 2000); mejoras en el rendimiento académico iguales o mejores que los generados por otros modelos, además, los estudiantes que participan en los proyectos tienen mayor responsabilidad por su propio aprendizaje que durante las actividades de aula más tradicionales (Boaler, 1999); oportunidades para desarrollar habilidades complejas, tales como el pensamiento de orden superior, resolución de problemas, colaboración y comunicación; acceso a una gama más amplia de oportunidades de aprendizaje en el aula, proporcionando una estrategia para involucrar estudiantes culturalmente diversos (Railsback, 2002).

Todo esto ha generado un gran interés por esta nueva estrategia instruccional y ha alcanzado su auge, en algunos casos se la impulsado desde las políticas públicas referentes a los sistemas educativos en algunos países. En el Ecuador, se han desarrollado convocatorias de proyectos impulsadas por instituciones gubernamentales en los que también participen los estudiantes. Esto ha hecho que las universidades también realicen este tipo de iniciativas y muchas de ellas ya han empezado a realizar "Proyectos Semilla", los cuales "son comunidades de aprendizaje y científicas en las que intervienen docentes y estudiantes a fin de contribuir al desarrollo de su competencia investigativa en el proceso de formación profesional que sirva de base al recambio/relevo generacional de investigadores de la comunidad científica universitaria" (Universidad de Guayaquil, 2015). 
Estos son proyectos que nacen en el aula de clase y que pueden ser presentados cuando la Universidad realiza una convocatoria interna. Luego es presentado y evaluado por un comité científico, tanto en la parte investigativa como presupuestaria hasta llegar a la fase de aprobación y desarrollo del mismo.

Por esta razón, se muestra en el presente trabajo un ejemplo de un proyecto semillero que surgió en el aula de un curso de tercer semestre de Física de una universidad del Ecuador con la finalidad de motivar a que sigan surgiendo iniciativas de este tipo.

\section{Métodos}

\section{Sujetos participantes}

Los participantes en el proyecto semillero son seis estudiantes de un curso de tercer semestre de Física: dos mujeres y cuatro hombres entre 19 y 22 años de edad, en conjunto con un profesor tutor.

\section{Primera sesión}

Se dedicó una sesión de clase para abordar las preguntas: ¿Existe algún problema con respecto a la enseñanza de la Física a nivel secundario? ¿Se lo puede identificar? ¿Se lo puede resolver?

Durante toda la sesión se dieron 15 minutos para contestarlo de forma individual, 10 minutos para dialogar en pareja con su compañero más cercano, 15 minutos para exponer los problemas en voz alta y finalmente 20 minutos para analizar si existía un problema común que podemos abordar juntos.

En esta sesión se identificó que la retroalimentación en las clases es muy difícil de realizar cuando el grupo de alumnos es grande y se delinearon algunas soluciones usando TICs.

\section{Segunda sesión}

Se dedicó una clase para abordar la siguiente pregunta: ¿Es posible diseñar algo (una herramienta o metodología) que sirva para resolver el problema de realizar una retroalimentación rápida a grupos grandes de alumnos?

Se dedicó 20 minutos a trabajos en grupos de 3 personas. Luego 20 minutos para que los grupos expongan sus soluciones. Finalmente 20 minutos para llegar a consensuar una única solución.

En esta sesión se identificó que mediante una página web que proporcione como recurso bancos de preguntas de opción múltiple (cuatro opciones y única respuesta) se puede brindar una solución óptima para retroalimentar durante la clase. También se formó el grupo de estudiantes interesados en proponer un proyecto semillero y se eligió al estudiante coordinador del mismo. 


\section{Tercera sesión}

Previo a esta sesión se envió a cada uno de los estudiantes participantes que leyeran el reglamento de semilleros de la universidad y los formatos que se debían llenar para presentar formalmente el proyecto. Además se les dio como plazo una semana de trabajo autónomo fuera del aula al grupo de estudiantes para que llenaran el formato y realizaran una presentación.

Finalmente, durante la sesión, el estudiante coordinador expuso frente a la clase la propuesta de proyecto mediante diapositivas. Al final de la exposición, el profesor tutor y los estudiantes realizaron preguntas o sugerencias para mejorar el proyecto. Todas estas sugerencias fueron consideradas para elaborar la versión final del semillero.

\section{Resultados}

A continuación se presenta, como resultado, una breve descripción del proyecto semillero que fue aprobado por la comisión científica de la universidad:

\section{Título del proyecto}

Aplicación multimedia para fortalecer el conocimiento general de la Física.

\section{Objetivo general}

Desarrollar una página web que permita la interacción entre estudiantes y tenga como fin fomentar el estudio y conocimiento de la Física.

\section{Objetivos específicos} específicos:

Para poder realizar el objetivo general antes descrito, se han desarrollado cinco objetivos

1. Implementar una base datos de preguntas basadas en los capítulos de Física de Bachillerato General Unificado.

2. Desarrollar una página web preliminar usando una muestra de banco de preguntas.

3. Realizar una prueba piloto de la página web con un mínimo de 100 usuarios.

4. Realizar las correcciones finales de la página web en base a sugerencias realizadas en la prueba piloto.

5. Comprobar mediante un grupo experimental y un grupo de control la efectividad de la página web en el rendimiento de los estudiantes.

Todo esto se lo realizará en el plazo de un año, empezando en marzo de 2016.

Para desarrollar la página web, se utilizara el software Hot Potatoes, el cual incluye seis aplicaciones, una de las cuales permite crear preguntas de opción múltiple para luego trasladarlos como página web. El programa fue desarrollado por el Humanities Computing and Media Centre 
de la Universidad de Victoria en Canadá; es gratuito, y es posible utilizarlo para cualquier propósito o proyecto (University of Victoria).

Por otra parte, las preguntas deben estar basadas en los capítulos de Física de Bachillerato General Unificado del Ecuador. Estas preguntas serán de opciones múltiples y una sola respuesta. Serán revisadas por un comité formado por el estudiante director del proyecto, el profesor tutor y un profesor externo, para asegurar la calidad y objetividad de las mismas.

\section{Resultados esperados e impacto del proyecto semillero}

El principal resultado es desarrollar una página web antes descrita que sirva como recurso para la enseñanza de la Física. Sin embargo, está página estará en mejora continua ya que se creará buzón electrónico de sugerencias administrado por los estudiantes que llevan el proyecto semillero.

Entre los beneficiarios directos estarían los estudiantes de Física que estén cursando el programa de Bachillerato general unificado en Ecuador. Según datos del Ministerio de Educación del Ecuador, en el 2012 se tenían alrededor de 500,000 estudiantes matriculados en Bachillerato en todo el Ecuador, de los cuales el $60 \%$ tiene acceso a internet en sus instituciones educativas (Ministerio de Educación del Ecuador, 2013). Es decir, serán alrededor de 300,000 estudiantes de entre 15 y 17 años de edad.

Por otra parte, se tienen beneficiarios indirectos, tales como padres de familia, profesores de Física e incluso universidades que impartan carreras relacionadas con la Física, ya que tentativamente puede crecer la demanda de estudiantes que decidan ingresar a este tipo de carreras.

Finalmente, teniendo en cuenta que la página web es virtual puede llegar a ser usado tanto a nivel nacional como internacional sin la necesidad de una fuerte inversión en publicidad.

Otros resultados internos del proyecto contemplan la publicación de dos artículos científicos o de revisión y una ponencia oral o en modalidad de póster.

\section{Conclusiones}

El ejemplo presentado anteriormente tiene como finalidad incentivar el uso de proyectos como una cuestión central, no periférica en el currículo o la planificación de estudios de las materias impartidas en el aula. Esta estrategia instruccional se centra en cuestiones o problemas en la que los estudiantes van al encuentro y debaten los conceptos centrales y los principios de la carrera que estudian (Stepien \& Gallagher, 1993). Estos proyectos tienen la capacidad de involucrar a los estudiantes en una investigación constructive (Bereiter \& Scardamalia, 1999) siempre y cuando se usen problemas reales, no escolarizados (Gordon, 1998). Esto dará como resultado que los estudiantes experimenten el aprendizaje de un todo integrado, en lugar de una serie de bloques separados a través de las horas del día. 
Sin embargo, es necesario mencionar que no es una labor que se limita a la enseñanza, sino también puede desembocar en un compromiso de la universidad con las comunidades locales, las familias y las empresas.

Esperamos que este trabajo inspire a más profesores para llevar el aprendizaje basado en proyectos en su clase, y que haya proporcionado ideas, consejos y herramientas para involucrar y desafiar a sus estudiantes.

\section{Bibliografía}

Bereiter, C., Scardamalia, M. (1999). Process and product in PBL research, Ontario Institutes for Studies in Education/University of Toronto.

Boaler, J. (1999). “Mathematics for the moment, or the millennium?”, Education Week. Available: http://www.edweek.org/ew/articles/1999/03/31/29boaler.h18.html

Bransford, J., Brown, A., Cocking, R. (2000). How people learn: Brain, mind, experience, and school, National Academy Press.

Gordon, R. (1998). Balancing real-world problems with real-world results, Phi Delta Kappan, pp. 390-393.

Jarrett, D. (1997). Inquiry strategies for science and mathematics learning, Northwest Regional Educational Laboratory.

Ministerio de Educación del Ecuador (2013). Ecuador: Indicadores Educativos 2011-2012, Ministerio de Educación del Ecuador.

Railsback, J. (2002). Project-based instruction: Creating excitement for learning, Northwest Regional Educational Laboratory.

Stepien, W., Gallagher, S. (1993). Problem-based learning: As authentic as it gets, Educational Leadership, vol. 51, pp. 25-28.

Thomas, J. (1998). Project-based learning: Overview. Buck Institute for Education.

Thomas, J. (2000). A review of research on project-based learning, Autodesk.

Universidad de Guayaquil (2015). "Reglamento provisional para el funcionamiento de semilleros de investigación de la UG". Available: http://www.dipa.ug.edu.ec/?p=1017

University of Victoria, “Hot Potatoes Home Page”, Available: https://hotpot.uvic.ca/ 